\title{
Rapid ventricular pacing for a basilar artery pseudoaneurysm in a pediatric patient: case report
}

\author{
Shahid M. Nimjee, MD, PhD, ${ }^{1,2}$ Tony P. Smith, MD, ${ }^{1}$ Ronald J. Kanter, MD, ${ }^{4}$ Warwick Ames, MD, ${ }^{3}$ \\ Kelly A. Machovec, MD, MPH, ${ }^{3}$ Gerald A. Grant, MD, ${ }^{2}$ and Ali R. Zomorodi, MD ${ }^{2}$ \\ Departments of ${ }^{1}$ Radiology and ${ }^{3}$ Anesthesiology, and Divisions of ${ }^{2}$ Neurosurgery and ${ }^{4}$ Pediatric Cardiology, Duke University \\ Medical Center, Durham, North Carolina
}

\begin{abstract}
Large cerebral aneurysms of the basilar apex are difficult to treat. Recently, endovascular treatment has mitigated much of the morbidity associated with treating these lesions. However, the morphology of aneurysms of the vertebrobasilar system can preclude endovascular treatment. Rapid ventricular pacing (RVP) facilitates open surgical treatment of cerebral aneurysms. It can assist in reducing the pressure of the neck of the aneurysm, allowing safe application of a clip. The authors present a case of a pediatric patient who developed a basilar artery pseudoaneurysm that required surgery. Given the large size of the aneurysm, RVP was performed, allowing the surgeons to dissect the dome of the aneurysm from the surrounding tissue and pontine perforating branches away from the lesion to safely clip the lesion. The patient had an uneventful recovery. To the authors' knowledge, this represents the first known case of RVP to aid in basilar artery clip occlusion in a pediatric patient.
\end{abstract}

http://thejns.org/doi/abs/10.3171/2014.11.PEDS14290

KEY WORDS rapid ventricular pacing; basilar artery aneurysm; pediatric; trauma; vascular disorders

$\mathrm{T}$ RAUMATIC cerebral aneurysms represent less than $1 \%$ of intracranial aneurysms and are typically associated with fractures of the skull base. These aneurysms have an unpredictable growth pattern, and the thin elastic walls of these lesions make them difficult to treat. The options include endovascular coil embolization, clip ligation via craniotomy, and bypass with parent vessel sacrifice. Endovascular coil embolization can result in recurrence of the aneurysm before thrombosis due to the weakness in the wall. This is of even greater concern in a younger patient population with a presumed longer lifespan. Open craniotomy with clip ligation of the aneurysm can be challenging due to arachnoid scarring resulting from the initial traumatic brain injury as well as the risk of rupture during vessel dissection and clip placement.

Rapid ventricular pacing (RVP) is a method to decrease vascular turgor while isolating the aneurysm and placing a clip on the neck. The technique has been used in intracranial aneurysm surgery in adults. Its use in the pediatric population has, to our knowledge, never been reported. We present the case of a 2-year-old boy who developed a traumatic basilar apex pseudoaneurysm after suffering a skull base fracture and traumatic brain injury due to a motor vehicle accident. We used RVP to facilitate vascular dissection and clip placement.

\section{Case Report}

History and Examination

A 2-year-old boy presented to the neurosurgery service for treatment of a traumatic basilar artery pseudoaneurysm. At the age of 14 months, he was involved in an unrestrained motor vehicle accident. On admission to the emergency department, he was intubated. A neurological examination revealed reactive pupils bilaterally. His brainstem reflexes were intact, and he moved all extremities purposefully.

The patient's only significant initial imaging study was a head CT scan, which revealed acute subarachnoid hemorrhage of the suprasellar, ambient, and quadrigemi-

ABBREVIATIONS MABP = mean arterial blood pressure; $\mathrm{PCA}=$ posterior cerebral artery; RVP = rapid ventricular pacing; $S C A=$ superior cerebellar artery SUBMITTED June 9, 2014. ACCEPTED November 12, 2014.

INCLUDE WHEN CITING Published online March 6, 2015; DOI: 10.3171/2014.11.PEDS14290.

DISCLOSURE The authors report no conflict of interest concerning the materials or methods used in this study or the findings specified in this paper. 
nal plate cisterns and minimally displaced longitudinal and transverse fractures of the right temporal bone with a longitudinal component also involving the right petrous apex and right aspect of the clivus. There were additional fractures of the right zygomatic process, left parietal bone, greater wing of the right sphenoid bone, and lateral right orbit; and diastasis of the left lambdoid suture and occipital base sutures.

A CT angiogram revealed a distal basilar artery aneurysm/pseudoaneurysm measuring $4 \times 2 \mathrm{~mm}$ (Fig. 1). The patient underwent repeat imaging, which revealed interval growth of the pseudoaneurysm to $7 \times 6 \mathrm{~mm}$ (Fig. 2).

The patient spent 10 weeks in the hospital recovering from his injuries. His postinjury sequelae included cortical blindness, hydrocephalus requiring a ventriculoperitoneal shunt, and status epilepticus, which was successfully treated with levetiracetam. Tracheostomy and percutaneous endoscopic gastrostomy tubes were placed, and the patient was discharged to a long-term rehabilitation facility. Neurological examination at hospital discharge demonstrated attention to verbal cues and smiling. He displayed intentional movements of the right upper and right lower extremities; there was only minimal movement on the left side.

The patient's follow-up visit included CT scanning and CT angiography of the brain, which showed a significant increase in the size of the basilar pseudoaneurysm to $21 \times$ $17 \mathrm{~mm}$ (Fig. 3). A diagnostic cerebral angiogram verified the findings (Fig. 4).

Given the rapid rate at which the aneurysm had grown, the neurosurgical team thought it imperative to treat the aneurysm. Preoperatively the neurological examination revealed that the boy was alert and smiling and in no acute distress. The patient's right pupil was sluggish to light, while the left pupil was fully reactive to light. He had good movement in his right upper and bilateral lower extremities. There was weakness in his left upper extremity. His sensation appeared intact.

\section{Treatment}

The patient was taken to the operating room for planned surgical clipping of the aneurysm. He was intubated without difficulty, and a bispectral index monitor was placed on his forehead. A left femoral arterial line was placed for intraoperative angiography. A 5-F right femoral venous sheath was placed, through which a 4-F multipole elec-
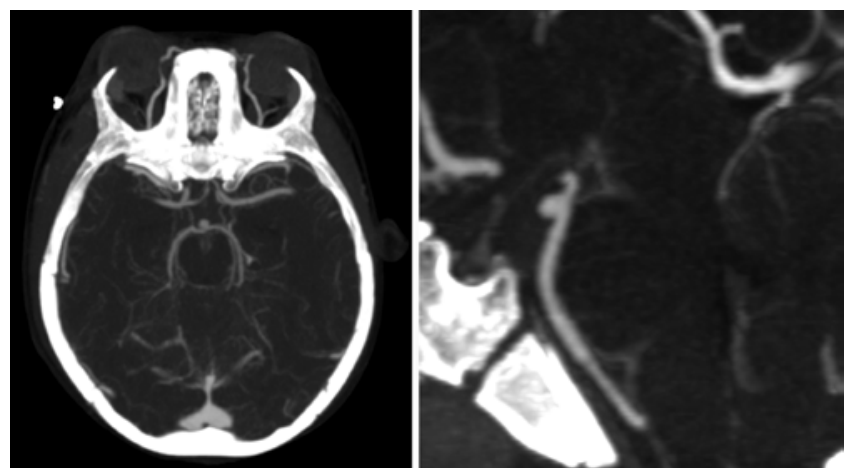

FIG. 1. Axial (left) and magnified sagittal (right) CT angiograms showing a pseudoaneurysm $4 \mathrm{~mm}$ in greatest diameter.
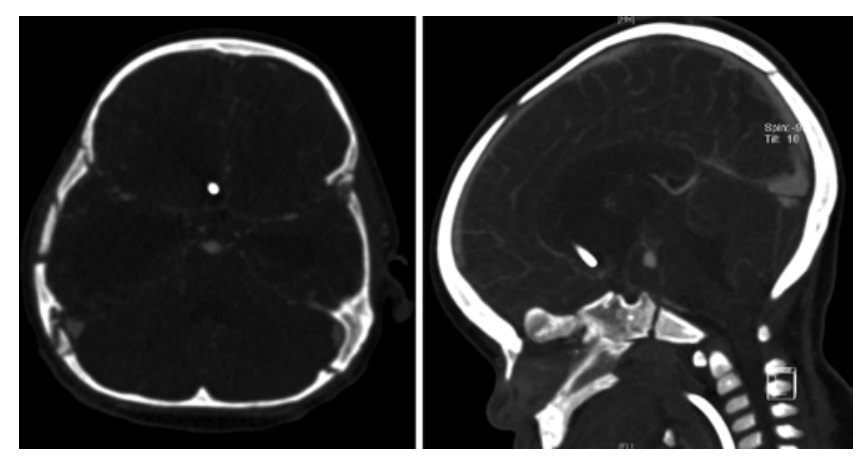

FIG. 2. Axial (left) and sagittal (right) CT angiograms showing growth of aneurysm after 4 weeks.

trophysiological catheter (St. Jude Medical) was passed. The catheter was advanced to the cardiac right ventricular apex under fluoroscopic guidance, and a ventricular pacing threshold of less than $1 \mathrm{~mA}$ was achieved at a $2-\mathrm{msec}$ pulse with the aid of a programmable cardiac stimulator (5328 Programmable Stimulator, Medtronic Inc.). Chest wall cutaneous defibrillation patches were placed for possible rescue defibrillation. High output pacing (5 mA at 10msec pulse width) was used to ensure ventricular capture at very rapid rates. During pacing and capture at $300 \mathrm{bpm}$, a desirable mean arterial blood pressure (MABP) of 23 $\mathrm{mm} \mathrm{Hg}$ was achieved. The patient was cooled passively to $33^{\circ}-34^{\circ} \mathrm{C}$. Motor and sensory evoked potential leads were placed to monitor brainstem activity. There was weakness on the left side, compatible with the patient's clinical baseline status.

The patient was then placed in a semilateral position with the head turned to the left in a Mayfield pin fixation system. A right reverse question mark incision was made. The temporalis muscle was carefully elevated out of the middle fossa. A zygomatic osteotomy was performed to allow the muscle to retract inferiorly.

A frontotemporal craniotomy was performed, followed by an extradural clinoidectomy. The dura was opened and tacked anteriorly. The brain appeared relaxed, which was aided by intraoperative mannitol and the patient's ventriculoperitoneal shunt.

The sylvian fissure was significantly scarred from the initial trauma but was carefully opened to separate the frontal and temporal lobes. The temporal lobe was el-
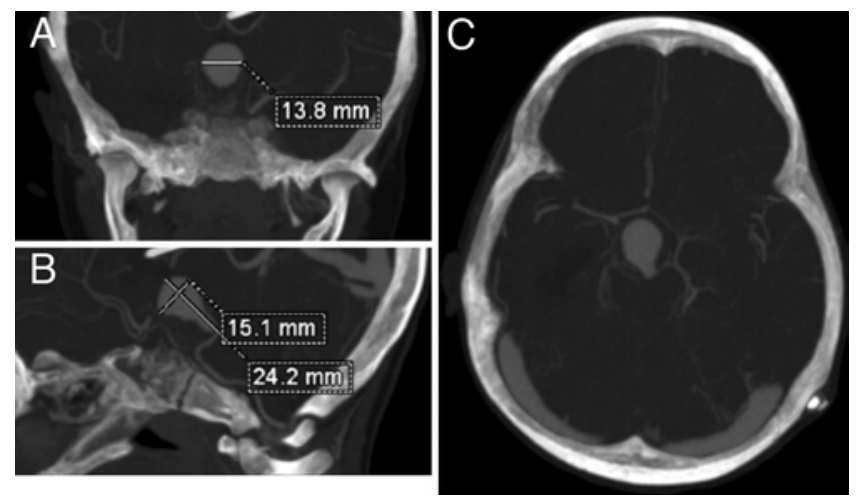

FIG. 3. Coronal (A), sagittal (B), and axial (C) CT angiograms showing marked enlargement of the aneurysm 32 weeks after initial imaging. 

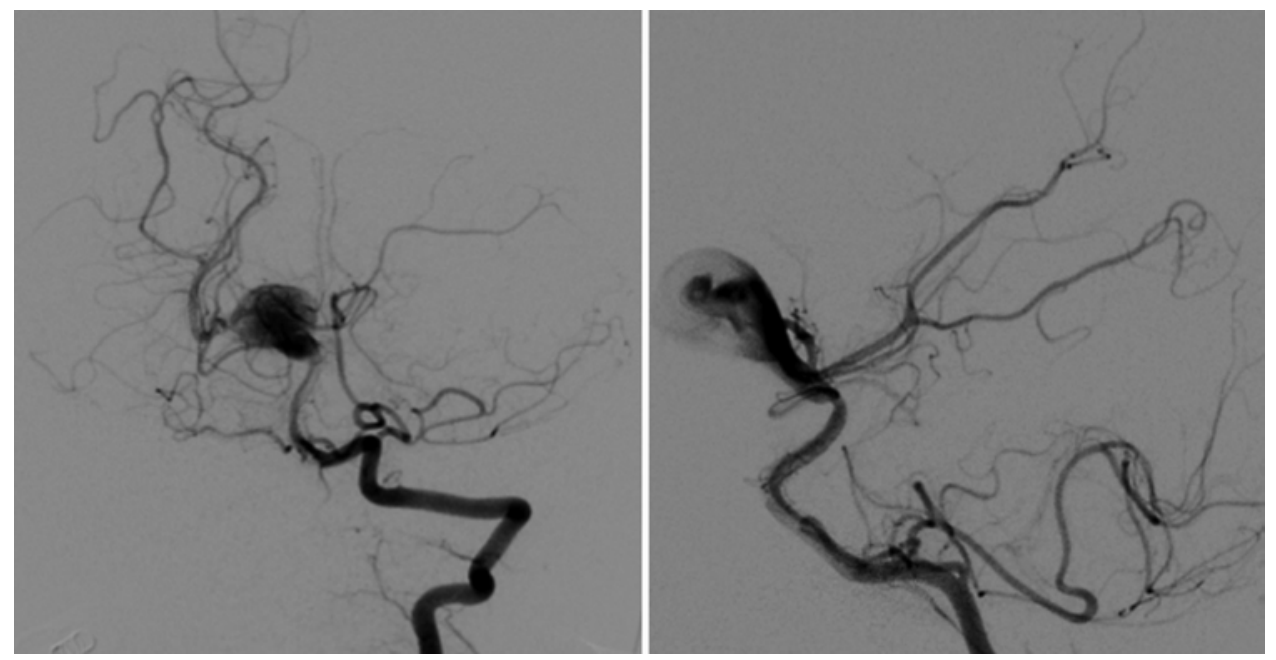

FIG. 4. Anteroposterior (left) and lateral (right) digital subtraction angiogram verifying aneurysm size, revealing a filling pattern consistent with thrombus within the aneurysm.

evated, and the edge of the tentorium was dissected to the incisure. The fourth cranial nerve was identified and protected. The third cranial nerve was identified and followed posteriorly. At one point the third cranial nerve was difficult to visualize, as it was tightly stretched over the dome of the aneurysm. To extend the proximal exposure, the trochlear and oculomotor canals were opened to the posterior edge of the cavernous sinus. The tentorium was then incised and tacked up. The aneurysm was identified but was difficult to dissect due to scarring from the initial trauma caused by the motor vehicle accident. The patient underwent RVP for 40 seconds, allowing the surgeons to dissect free the dome of the aneurysm and safely dissect pontine perforators away from the aneurysm. This was repeated 4 more times with the length of pacing ranging from 34 to 45 seconds. The patient tolerated RVP, returning to sinus rhythm and baseline MABP levels spontaneously. The repetitive cycles of RVP enabled dissection of the aneurysm to the level of the basilar artery. The surgeons identified the normal basilar artery, proximal to the dome of the aneurysm. The patient underwent a fifth RVP for 74 seconds, allowing the surgeons to dissect the posterior cerebral arteries (PCAs), visualize the right $\mathrm{P}_{1}$ and $\mathrm{P}_{2}$ segments, and identify both superior cerebellar arteries (SCAs). Of note, the wall of the aneurysm was very thin with translucent areas, consistent with a pseudoaneurysm. After extensive dissection made possible by RVP, a clip was placed across the base of the aneurysm.

The clip was then repositioned with the aid of RVP for 55 seconds. Sinus rhythm and MABP returned to baseline spontaneously. An intraoperative angiogram revealed less flow compared with the previous angiogram. The large size of the aneurysm made PCA and SCA visualization difficult, and, thus, the aneurysm was opened and the clot within the aneurysm was resected with the aid of a Cavitron Ultrasonic Aspirator (CUSA). The clip was repositioned one last time, utilizing RVP for 67 seconds. Ventricular fibrillation occurred and sinus rhythm returned after electric defibrillation. With the aid of an intraoperative Doppler ultrasound probe, the surgeons verified bilateral PCA flow of the $\mathrm{P}_{1}$ and $\mathrm{P}_{2}$ segments as well as SCA flow. Hemostasis was obtained, the dura was closed, and the bone was replaced with absorbable plates. The zygoma was secured with screws, and the temporalis and fascia were elevated to the superior temporal line. The wound was then closed in layers.

\section{Postoperative Imaging and Follow-Up}

The patient was taken to the pediatric intensive care unit intubated given the length of the case; he was extubated the next day. Examination on postoperative Day 2 revealed an alert patient who was smiling and in no acute distress. His pupillary examination was stable, with a sluggishly reactive right and fully reactive left pupil. His motor examination revealed stable left upper-extremity weakness. A CT scan of his brain obtained 24 hours after surgery showed clip placement with no evidence of cerebral infarction (Fig. 5). At his 1-month follow-up, the patient's left upper-extremity strength had improved, which was now grossly equal to his right upper extremity. All other neurological findings were stable from his immediate postoperative examination. At the 12-month follow-up, the patient was ambulating independently with grossly equal bilateral upper-extremity strength. A postoperative angiogram demonstrated a broad base at the confluence of the basilar artery and 2 PCA arteries without aneurysm recurrence (Fig. 6).

\section{Discussion}

To our knowledge, this is the first published case report of a traumatic intracranial pseudoaneurysm in a pediatric patient treated surgically with the assistance of RVP. Traumatic aneurysms represent less than $1 \%$ of all intracranial aneurysms. ${ }^{6}$ The prevalence of pediatric intracranial aneurysms is $1 \%-2 \%$, of which $30 \%$ are traumatic., ${ }^{1,46}$ Basilar skull fractures are associated with traumatic aneurysms of the basilar artery, as was the case with our patient. ${ }^{1}$ The growth of traumatic intracranial aneurysms is unpredictable; therefore, additional imaging is essential in deciding when to treat this lesion. ${ }^{3}$ Our patient was diagnosed with a basilar artery pseudoaneurysm measuring $4 \times 2 \mathrm{~mm}$ on 


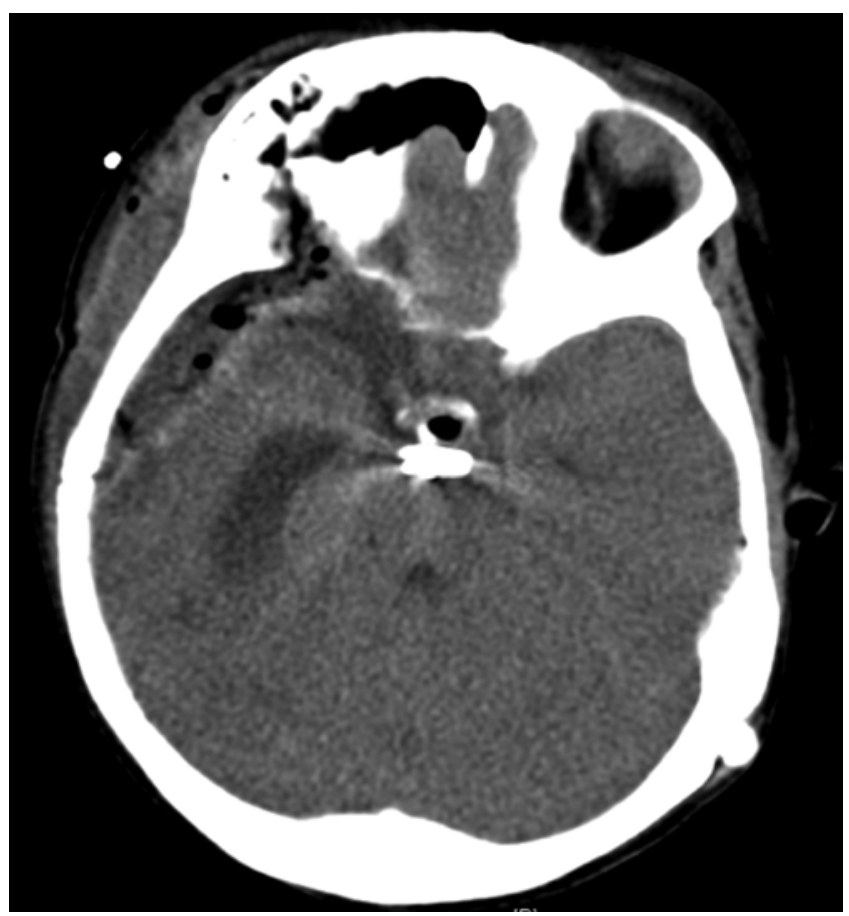

FIG. 5. Brain CT scan showing clip placement and no evidence of cerebral infarction.

initial imaging that grew to $21 \times 17 \mathrm{~mm} 32$ weeks later. The reported time to hemorrhage of traumatic aneurysms from the time of initial trauma is 21 days and carries a mortality rate as high as $50 \%$. $^{3,6}$ Due to the significant increase in growth, treatment became a priority.

The 3 main methods to treat traumatic aneurysms include endovascular coiling, open clip ligation, and vessel bypass. . $3,6-9,12$ In the pediatric population and traumatic aneurysms, arguments supporting clip ligation include that it represents definitive treatment of the aneurysm, allows for reconstruction of the parent vessel, and alleviates mass effect of the aneurysm by thrombectomy. ${ }^{4,6}$ We chose open surgery for these very reasons. Moreover, we also postulated that the wall of the pseudoaneurysm was thin and coiling would risk rupture. Our hypothesis proved to be true when we saw the aneurysm wall intraoperatively. Since the development of Guglielmi coils, there are reports of treating traumatic intracranial aneurysms using endovascular techniques. 3,7,9,12 There is, however, a risk of recurrence, necessitating retreatment multiple times. ${ }^{3,12}$

Given the large size of the aneurysm, history of trauma resulting in significant scarring, and the importance of preserving small but crucial pontine perforating vessels, safe dissection of the aneurysm was imperative. To facilitate such dissection, we used RVP. While not a novel concept in neurosurgery, this is the first report of it being used in a pediatric patient. The first use of RVP in aneurysm surgery was reported in 1966, where pacing wires were advanced into the right ventricle via endovascular placement from the arm and were used to generate near circulatory arrest to facilitate intracranial aneurysm clip ligation. ${ }^{15}$ RVP fell out of fashion for years, largely replaced by hypothermic arrest. More recently, the method has been described as a safe and effective technique to fa-

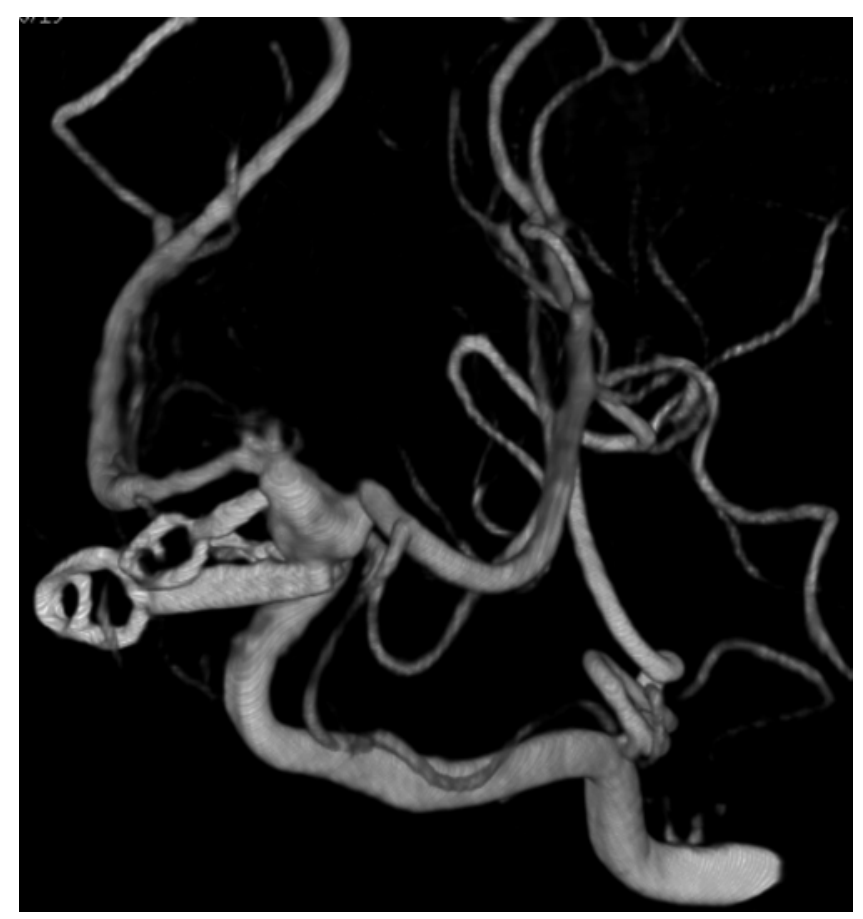

FIG. 6. 3D reconstruction of a digital subtraction angiogram demonstrating placement of both aneurysm clips around the aneurysm. There is a broad base at the confluence of the basilar artery and 2 PCAs without evidence of aneurysm recurrence.

cilitate cerebral aneurysm clip ligation and arteriovenous malformation resection. ${ }^{14,17}$

Adenosine-induced cerebral hypotension is another method to temporarily reduce vessel tension in cerebrovascular surgery. Popularized in 2005, this technique effectively induces transient hypotension and facilitates dissection and clip ligation of intracranial aneurysms. ${ }^{5}$ This method, however, has risks that limit its use. There are general guidelines for dosing in adults, but the doses can vary, resulting in unpredictable dose responses in patients. In our case, there were no guidelines for pediatric patients, and RVP offered tighter control of the duration of hypotension. ${ }^{14}$ Contraindications of using adenosine include chronic obstructive pulmonary disease, coronary artery disease, and cardiac conduction abnormalities. ${ }^{5}$

There are risks associated with RVP, chief among them are arrhythmias, including ventricular tachycardia and fibrillation. ${ }^{10,13,16}$ These are treated by override pacing or, in our case, defibrillation. Of note, while we used RVP multiple times throughout the case, the patient only required defibrillation once and was otherwise able to spontaneously convert to sinus rhythm. There is also risk of catheter placement resulting in cardiac perforation with subsequent cardiac tamponade, although it is rare. ${ }^{11}$

\section{Conclusions}

This novel case report demonstrates that the use of RVP is a safe and effective method to treat difficult intracranial aneurysms in pediatric cerebral aneurysm surgery and should be considered by surgeons and anesthesiologists who encounter complex cerebrovascular cases. 


\section{References}

1. Allison JW, Davis PC, Sato Y, James CA, Haque SS, Angtuaco EJ, et al: Intracranial aneurysms in infants and children. Pediatr Radiol 28:223-229, 1998

2. Bowers C, Riva-Cambrin J, Couldwell WT: Efficacy of clip-wrapping in treatment of complex pediatric aneurysms. Childs Nerv Syst 28:2121-2127, 2012

3. Fulkerson DH, Voorhies JM, McCanna SP, Payner TD, Leipzig TJ, Scott JA, et al: Endovascular treatment and radiographic follow-up of proximal traumatic intracranial aneurysms in adolescents: case series and review of the literature. Childs Nerv Syst 26:613-620, 2010

4. Kakarla UK, Beres EJ, Ponce FA, Chang SW, Deshmukh VR, Bambakidis NC, et al: Microsurgical treatment of pediatric intracranial aneurysms: long-term angiographic and clinical outcomes. Neurosurgery 67:237-250, 2010

5. Khan SA, Nimjee SM, Guinn NN, Zomorodi AR, Lam AM, Britz GW, et al: The use of adenosine in cerebral aneurysm clipping: a review. Curr Anesthesiol Rep 3:210-213, 2013

6. Larson PS, Reisner A, Morassutti DJ, Abdulhadi B, Harpring JE: Traumatic intracranial aneurysms. Neurosurg Focus 8(1):E4, 2000

7. Mahmoud M, Roshdi E, Benderbous D: Traumatic pseudoaneurysms of the common carotid and vertebral artery in a four-year-old child. Interv Neuroradiol 18:348-352, 2012

8. Mao Z, Wang N, Hussain M, Li M, Zhang H, Zhang Q, et al: Traumatic intracranial aneurysms due to blunt brain injury-a single center experience. Acta Neurochir (Wien) 154:2187-2193, 2012

9. Medel R, Crowley RW, Hamilton DK, Dumont AS: Endovascular obliteration of an intracranial pseudoaneurysm: the utility of Onyx. J Neurosurg Pediatr 4:445-448, 2009

10. Mehta C, Desai T, Shebani S, Stickley J, DE Giovanni J: Rapid ventricular pacing for catheter interventions in congenital aortic stenosis and coarctation: effectiveness, safety, and rate titration for optimal results. J Interv Cardiol 23:7 13,2010

11. Nashed G, French B, Gallagher D, Hopkins A, Juergens C, Leung D, et al: Right ventricular perforation with cardiac tamponade associated with use of a temporary pacing wire and abciximab during complex coronary angioplasty. Catheter Cardiovasc Interv 48:388-389, 1999

12. Quintana F, Diez C, Gutierrez A, Diez ML, Austin O,
Vazquez A: Traumatic aneurysm of the basilar artery. AJNR Am J Neuroradiol 17:283-285, 1996

13. Sack S, Kahlert P, Khandanpour S, Naber C, Philipp S, Möhlenkamp S, et al: Revival of an old method with new techniques: balloon aortic valvuloplasty of the calcified aortic stenosis in the elderly. Clin Res Cardiol 97:288-297, 2008

14. Saldien V, Menovsky T, Rommens M, Van der Steen G, Van Loock K, Vermeersch G, et al: Rapid ventricular pacing for flow arrest during cerebrovascular surgery: revival of an old concept. Neurosurgery 70 (2 Suppl Operative):270-275, 2012

15. Small JM, Stephenson SC, Campkin TV, Davison PH, McIlveen JS: Elective circulatory arrest by artificial pacemaker. Lancet 287:570-572, 1966

16. Webb JG, Pasupati S, Achtem L, Thompson CR: Rapid pacing to facilitate transcatheter prosthetic heart valve implantation. Catheter Cardiovasc Interv 68:199-204, 2006

17. Whiteley JR, Payne R, Rodriguez-Diaz C, Ellegala DB, Reeves ST: Rapid ventricular pacing: a novel technique to decrease cardiac output for giant basilar aneurysm surgery. J Clin Anesth 24:656-658, 2012

\section{Author Contributions}

Conception and design: Nimjee, Smith, Grant, Zomorodi. Acquisition of data: Nimjee, Smith, Kanter, Ames, Zomorodi. Analysis and interpretation of data: Drafting the article: Nimjee. Critically revising the article: all authors. Reviewed submitted version of manuscript: Nimjee, Machovec, Grant, Zomorodi. Approved the final version of the manuscript on behalf of all authors: Nimjee.

\section{Supplemental Information}

\section{Current Affiliation}

Dr. Nimjee: Department of Neurological Surgery, The Ohio State University Wexner Medical Center, Columbus, $\mathrm{OH}$.

\section{Correspondence}

Shahid M. Nimjee, Department of Neurological Surgery, The Ohio State University Wexner Medical Center, N-1014 Doan Hall, 410 W. 10th Ave., Columbus, OH 43210. email: shahid. nimjee@osumc.edu. 